\title{
C -292
}

09. STRUCTURES OF ORGANIC, ORGANOMETALLIC AND COORDINATION COMPOUNDS

09. 3-10 STRUCTURAL PROPERTIES OF $R_{2} P_{2} P_{2} P_{2}$-BRIDGED COMPLEXES OF PALLADIUM AND PLATINUM. BY Lj. Manojlović-Muir and K.W. Muir, Department of

Chemistry, University of Glasgow, Glesgow GI2 8QQ, U.K.

Complexes of palladium and platinum stabilised by bridging $R_{2} \mathrm{PCH}_{2} \mathrm{PR}_{2}$ ligands display diverse and often novel structural properties, including several modes of metal-metal interactions. Their molecular". structures are systematically studied in our laboratory by diffraction techniques. The object is to investigate the role of the $\mathrm{R}_{2} \mathrm{PCH}_{2} \mathrm{PR}_{2}(\mathrm{R}=\mathrm{Ph}$ or $\mathrm{Me})$ ligands in promoting rich and versatile reactivity of these complexes and elucidate the interplay between electronic and steric demands of the metal centres, steric flexibility of the bridging phosphines and conformational changes of the $\mathrm{M}_{2} \mathrm{P}_{2 n} \mathrm{C}_{n}$ dimetallacycles. New results on homo- and hetero-bimetallic and polymetallic complexes in which the oxidation state of the metal atom changes from 0 to +4 wiIl be shoun. being hetero-atoms $(N, 0)$ or $\pi$-bonds, and $s i x$ systems of type (3) with $D=1,2-b$ is (dimethylamino) ethane.

(2) is analogous to the diborane molecule, i.e. it is stabilized by two 3 -centre bonds, for which localized carbanion orbitals $(x=2$ or 3$)$ must be available. One of these complexes has the point symmetry 2 , another one approximately 2 and the remaining three 1 . Two of the structures contain allenyl groups which offer a spa orbital for the 3-centre bond, while their asystem acts as a donor $D$.

If the carbanion charge is $\pi$-delocalized, type (2) structures are not possible. Instead the DDLi moiety can be $\pi$-bonded to the carbanion in the fashion (3). The position of this $\mathbb{a}$-bonding on the carbanion surface shows where the $L i$ finds sufficient charge and optimal overlap. This is not only a function of charge delocalization but a Iso of conformation of the anion. The six systems of type (3) contain dianions. Thus, a second DDLi group is $\boldsymbol{\pi}$-bonded at the opposite $\pi$-surface of the dianion. Three of the structures have an approximate symmetry 2 .

All phenyl groups involved show a characteristical deformation pattern of the bond angles within the ring suggesting an increased weight of a p-quinoid resonance structure. This is plausible, since the $C-C$ bond connecting the ring to the chain has increased bond order and some of the charge is transferred to the (peripheral) p-position.

09. 3-12 NEUTRON DIFFRACTION STUDY OF THE C-H-Ti INTERACTION IN TiMeCl $1_{3}$ (dmpe).* A. 3. Schultz and J. M. Wi 11 iams, Argonne National Laboratory, Argonne, Illinois 60439, USA: T. F. Koetzle, Brookhaven National Laboratory, Upton, New York 11973, USA: Z. Dawoodi, M. L. H. Green and K. Prout, University of Oxford, Oxford OXI $30 R$, U.K.

Previous neutron diffraction studies using conventional and time-of-flight techniques have demonstrated the existence of stable 3-center, 2-electron $\mathrm{C}-\mathrm{H}-\mathrm{met} a \mathrm{l}$ bonds in cases where the carbon atom is not directly bonded to the metal (Schultz, et al., Science $(1983), 220,197)$. For interactions involving a-carbon and a-hydrogen atoms in Ta-neopentylidene complexes (Schultz, Brown, Willjams and Schrock, J. Amer. Chem. Soc. (1981), 103, 169), viz., $\left[\mathrm{Ta}\left(\mathrm{CHCMe}_{3}\right)\left(\mathrm{PMe}_{3}\right) \mathrm{Cl}_{3}\right]_{2}$ and $\mathrm{Ta}\left(\mathrm{CHCMe}_{3}\right)\left(\mathrm{C}_{5 \mathrm{Me}}\right)\left(\mathrm{C}_{2} \mathrm{H}_{4}\right)-$ (РMe3), the $\mathrm{Ta}-\mathrm{C}_{\alpha}-\mathrm{C}_{\beta}$ angles approach $180^{\circ}$, the $\mathrm{Ta}_{-} \mathrm{C}_{\alpha}-$ $H_{\alpha}$ angles are less than $90^{\circ}$ and the $\mathrm{C}_{\alpha}-\mathrm{H}_{\alpha}$ bonds are elongated (1.13A). These interactions probably involve the 2 electrons in the $\mathrm{C}-\mathrm{H}_{\mathrm{H}}$ bond and a metal dir-orbital. Two common features of the Taneopentylidene structures are the presence of only one a-hydrogen atom and the absence of B-hydrogen atoms. We have now collected single crystal neutron diffraction data at the Brookhaven HFBR on the title compound at $20 \mathrm{~K}$. A previous $x$-ray study (Dawoodi, Green, Mtetwa and Prout, J. Chem. Soc., Chem. Commun. (1982), 1410) indicated an interaction involving one of the three $\alpha$-hydrogen atoms on the methyl ligand. The results of an analys is of the low temperature neutron data will be described in detail.

*Me $=\mathrm{CH}_{3} ;$ dmpe $=\mathrm{Me}_{2} \mathrm{PCH}_{2} \mathrm{CH}_{2} \mathrm{PMe}_{2}$.

Work supported in part by the J.S: Department of Energy.
(1)

(2)
$5 p^{x}$ delocal

of carbanion

(3) 